Manuelle Medizin 2011 · 49:301-302

DOI 10.1007/s00337-011-0867-2

Online publiziert: 30. September 2011

(c) Springer-Verlag 2011

\author{
W. v. Heymann \\ Bremen
}

\title{
FIMM und ESSOMM - Impulse auch für Deutschland
}

praktoren und die Osteopathen wird dieses Schriftstück jedoch nicht eingereicht (nebenbei gesagt würde die Anerkennung durch die WHO mindestens 100.00o USDollar kosten), sondern dient eher dazu, die FIMM-Gesellschaften und ihre Positionen gegenüber den Anbietern von Gesundheitsleistungen bekannt zu machen. Ich möchte schon jetzt alle gesundheitspolitisch interessierten Manualmediziner dazu aufrufen, die Mitte Oktober zu beschließende Version dieser Erklärung zu diskutieren und zu kommentieren, damit es bald eine in allseitigem Konsens gefasste Endversion geben kann. Die jeweils aktuelle Version (heute: 1.6o) ist über die Geschäftsstelle der DGMM zu erhalten. Mitwirkung ist dringend erwünscht!

Eine wichtige Funktion kommt auch dem englisch-französisch-deutschen Glossar-Komitee der Ausbildungs-Kommission zu, welche ebenfalls in Prag tagen wird. Hier müssen die unterschiedlich gebrauchten Begriffe aus der Welt der manuellen Medizin sprachlich analysiert und so definiert werden, dass eine gegenseitige Verständigung möglich wird. Wer kann denn schon wissen, dass mit dem von den amerikanischen Osteopathen verwendete Begriff „philosophy“ mitnichten eine eigene Weltanschauung im Sinne eines Aristoteles oder Hegels gemeint ist, sondern am ehesten durch den deutschen Begriff Konzept zu übersetzen ist?

Wer immer eine solche Begriffserklärung herbeiführen will, ist ebenfalls herzlich willkommen, sein Problem darzulegen - am besten an das deutsche GlossarMitglied Mathias Psczolla.
Europa steht aus manualmedizinischer Sicht unter dem Einfluss des Bologna-Prozesses für die PostgraduiertenWeiterbildung und der Politik der UEMS (Union Européenne des Médecins Spécialistes - Verband der europäischen Fachärzte). Hier hat sich - auch angesichts der seit Langem sehr inaktiven frankophonen UEMMA - in den letzten 3 Jahren ein Zusammenschluss mit dem $\mathrm{Na}$ men ESSOMM (European Scientific Society for Manual Medicine - wissenschaftliche europäische Gesellschaft für Manuelle Medizin) gebildet. Sie hat durch intensive Arbeit auf verschiedenen Ebenen endlich erreicht, die manuelle Medizin am 6./7. Oktober in Neapel dem Vorstand der UEMS vorstellen zu können, um durch ein Multidisciplinary Joint Committee (MJC) der manuellen Medizin den Status einer europaweit anerkannten Zusatzbezeichnung zu ermöglichen. Dies wäre dann ein sehr großer Erfolg für die internationale manuelle Medizin.

Um die europäischen Gesellschaften der manuellen Medizin für das Postgraduierten-Kapitel des Bologna-Prozesses fit zu machen, hat die ESSOMM ihren ersten Instruktorenkurs in Rom abgehalten. Sehr wenige europäische Gesellschaften erfüllen heute die ärztliche Weiterbildung der Manualmediziner mit einem Kurrikulum von 30 ECTS-Credits. Wenn man davon ausgeht, dass für einen ECTS-Credit 15 bis 20 Stunden Unterricht veranschlagt werden, müssen auch die deutschen Manualmediziner mit derzeit 320 Stunden noch ein Menge an Kleingruppen- und Eigenstudium nachweisen, um 500 bis 
700 Stunden zu erfüllen. Immerhin haben dieses Jahr schon 5 Gesellschaften Delegierte entsandt, um den Prozess der Standardisierung der ärztlichen Weiterbildung auf europäischem Niveau in Gang zu setzen. Hier werden jedoch weitere Anstrengungen erforderlich sein; weitere nationale Gesellschaft müssen in diesen Prozess einheitlicher Standards und Prozessqualität einbezogen werden.

Um schließlich neben der Gesundheitspolitik und der Weiterbildung auch noch die wissenschaftlichen Grundlagen anzusprechen, werden demnächst in einer Reihe von Artikeln die Ergebnisse und Schlussfolgerungen einer äußerst intensiven ESSOMM-Tagung veröffentlicht. Analog den Inhalten dieser Tagung sollen Artikel zu den folgenden Themen erscheinen: Neuroanatomie, Gelenkmechanik, Schmerzhemmung, rezeptive Felder, motorische Reflexantwort, psychische Aspekte des Schmerzes und autonomes Nervensystem. Wir dürfen gespannt sein auf diese Ergebnisse der von den ESSOMMMitgliedern SAMM, MWE und ÄMM abgehaltenen dritten Bodenseekonferenz.

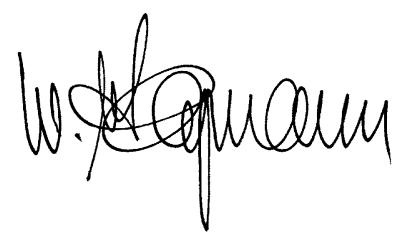

W. v. Heymann

Vizepräsident der FIMM

\section{Korrespondenzadresse}

\section{Dr. W. v. Heymann}

Schwachhauser Heerstr. 367, 28211 Bremen

heymann@cosit.de

\section{Jahre Carol-Nachman-Preis für Rheumatologie der Landeshauptstadt Wiesbaden}

\section{Die Spielbank Wiesbaden stiftet 2012 im 40. Jahr einen Preis zur Förderung der klinischen, therapeutischen und experimentellen Forschungsarbeit auf dem Gebiet der Rheumatologie.}

Der Carol-Nachman-Preis für Rheumatologie wird jährlich von der Landeshauptstadt Wiesbaden verliehen für hervorragende innovative Forschungsarbeiten auf dem Gebiet der Rheumatologie.

Der mit 37.500 Euro dotierte Preis gehört zu den höchst dotierten Medizinpreisen Deutschlands. Carol-Nachman-Preis und -Medaille tragen den Namen ihres Stifters, des langjährigen Spielbank-Konzessionärs und Wiesbadener Ehrenbürgers, Carol Nachman. Seit 1972 wird der Preis an international renommierte Wissenschaftlerinnen und Wissenschaftler vergeben.

Eingereicht werden können publizierte Arbeiten (auch kumulativ) zu einem wissenschaftlichen Fokus der vergangenen Jahre im Original und zusammenfassend dargestellt.

\section{Arbeiten für 2012 können bis zum}

15. Dezember 2011 in 10-facher Ausfertigung eingereicht werden .

Nähere Auskünfte erhalten Sie bei der Vorsitzenden des Carol-NachmanKuratoriums:

Prof. Dr. med. Elisabeth Märker-Hermann HSK Dr.-Horst-Schmidt-Kliniken GmbH Ludwig Erhard Str. 100 65199 Wiesbaden, Deutschland Tel: +49 (0)611/43-9777 Fax: +49 (0)611/43-9779 E-Mail: elisabeth.maerker-hermann@ hsk-wiesbaden.de
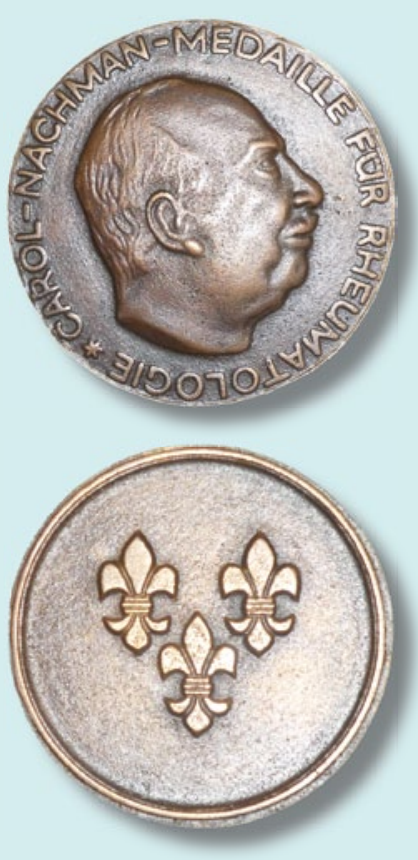

Kuratorium

des Carol-Nachman-Preises:

- Prof. Dr. Elisabeth Märker-Hermann (Vorsitzende)

- Prof. Dr. Joachim R. Kalden (Ehrenvorsitzender)

- Detlev Bendel

- Dr. med. Wolfgang W. Bolten

- Prof. Dr. Jürgen Braun

- Prof. Dr. Steffen Gay

- Prof. Dr. Joachim Grifka

- Prof. Dr. Thomas W.J. Huizinga

- Prof. Dr. Andreas Schwarting

- Prof. Dr. Joachim Sieper

- Henning Wossidlo 